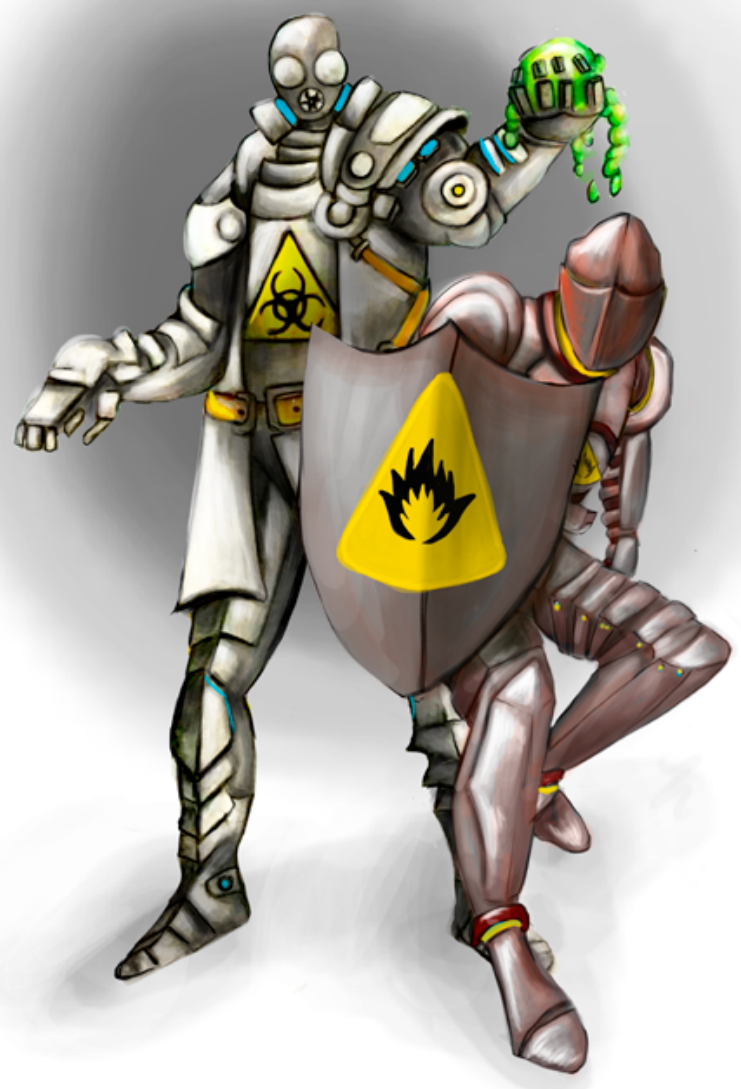

- Capítulo 2 -

\title{
Desarrollo del laboratorio para el análisis de los riesgos: Biológico, Químico y de Seguridad
}

\section{Autores}

María Alexandra Malangón Torres. Fisioterapeuta, Magister en Prevención de Riesgos Laborales. Docente en instituciones de educación superior en el área de ergonomía, medicina preventiva del trabajo y prácticas empresariales 
en todo lo relacionado con el Sistema de Gestión de Seguridad y Salud en el trabajo. Consultora Empresarial en Ergonomía para ARL.

Correspondencia:mmalagon@poligran.edu.co

Nohora Ramos Duarte. Microbióloga Industrial, Máster en Prevención de Riesgos Laborales Universidad de la Rioja España. Experiencia en la dirección de programas de Gerencia Integral del Riesgo Seguridad y Salud en el trabajo, Directiva de programas posgraduales de SST, desempeño dentro del área de seguridad y docencia universitaria por un periodo de 10 años, coordinadora de prácticas del programa de pregrado y asesora en la construcción de cursos y programas en modalidad presencial y virtual, en el área de Seguridad y Salud en el trabajo.

Correspondencia:nramosdu@poligran.edu.co

\section{Resumen}

La búsqueda de un escenario de práctica en el análisis de riesgos laborales para riesgos de seguridad, biológicos y químicos condujo a los autores al diseño, planeación y elaboración de un simulador que cumple el papel de un laboratorio virtual para que los estudiantes tengan escenarios de práctica que fortalezcan los conocimientos adquiridos en su proceso de formación profesional y les permita a los tutores evidenciar que realmente han adquirido las habilidades necesarias para enfrentarse a su quehacer profesional. Este nuevo tipo de estrategias pedagógicas basadas con la elaboración de una herramienta de software pensada desde las necesidades de los estudiantes en las aulas propende por la calidad académica en programas de modalidad virtual, en este caso. El proceso que se describe en este documento evidencia como el profesional en Seguridad y salud laboral a través del empleo de una herramienta de simulación desarrollada por los autores y sustentada en una estrategia pedagógica definida, puede realizar una identificación efectiva de los peligros, valorar los riesgos y mitigarlos evitando la presencia de enfermedades, accidentes o incidentes generados por la no prevención de los riesgos en un ambiente laboral para riesgo biológico, químico y de seguridad. Para esto se incluyeron dentro del simulador tres métodos diferentes específicos 
para cada uno de los riesgos seleccionados, de tal manera que el estudiante pueda proponer estrategias intervención optimas según el caso y próximas a una realidad laboral. La estrategia de aprendizaje está pensada para que el estudiante vaya más allá del análisis de una empresa y se apropie de una metodología de análisis que pueda aplicar en cualquier tipo de organización.

\section{Palabras claves}

Seguridad y salud en el trabajo, identificación y evaluación de riesgos, desarrollo de competencias, análisis de peligros, ambiente de trabajo.

\section{Introducción}

El estudio de los riesgos se puede considerar como una actividad sistemática que tiende a perfeccionarse buscando la minimización de los accidentes laborales, enfermedades profesionales y las pérdidas materiales generadas por la interacción de factores desfavorables que incrementan la probabilidad de que nuestra población productiva se vea afectada.

Cuando se habla de la identificación y posible evaluación de los riesgos a los que están expuestos los trabajadores, se requiere que el profesional desarrolle un pensamiento crítico-analítico frente a cualquier situación para que sea capaz de interpretar las situaciones nocivas en el trabajo y tomar decisiones para proponer la mejor intervención. Para desarrollar estas habilidades, se debe ofrecer a los estudiantes ambientes de aprendizaje propicios que les permitan la adquisición de las competencias. De esta manera, el estudiante puede implementar programas, modificar procesos y procedimientos, proponer actividades de prevención de enfermedades laborales y control o minimización de los accidentes de trabajo.

Una estrategia pedagógica altamente efectiva que confronta al estudiante con los conocimientos adquiridos y permiten evidenciar la adquisición de la competencia es como lo menciona (Gómez, Gómez y González, 2007) el aprender haciendo (learning-by-doing) que promueve un aprendizaje experiencial, que se aplica en procesos educativos donde el estudiante debe enfrentarse a situaciones reales como pilotar un avión o controlar una central nuclear. Sin embargo, en esos casos los sistemas que se manejan son costosos y pueden poner en riesgo vidas humanas por dejarlo en manos inexpertas. Los 
simuladores solucionan el problema porque recrean situaciones en las que el estudiante actúa como si se encontrara en el mundo real, desarrollando las habilidades que necesita en un entorno seguro y controlado.

El reto es construir situaciones próximas a la realidad laboral que confronten al alumno y pueda aplicar tanto los conceptos como las metodologías existentes para la identificación y trabajo de los riesgos de diversa índole. En lo concerniente a los riesgos laborales, el estudiante debe hacer un análisis no solo como práctica de la teoría vista en los módulos, sino que debe aplicar de manera integral y transversal sus conocimientos adquiridos, a esto se suman los que ha aprendido en el transcurso de la simulación. Por tal razón, estos ambientes simulados son ideales para los alumnos de modalidad virtual porque pueden emular todo un proceso y evidenciar la adquisición de las competencias con tan solo una conexión a internet puesto que en muchos casos el desplazamiento hacia una empresa real es limitado y complejo.

Para esta experiencia de aprendizaje se abordaron los riesgos de tipo biológico, químico, y de seguridad. Para los tres se diseñó una estrategia pedagógica transversal basada en aprendizaje situacional y basado por proyectos, sin embargo, para la evaluación de estos se emplearon tres metodologías propias para cada riesgo: Biológico (Biogaval), Químico (Gatiso del benceno) y de seguridad (GTC-45). Este documento registra todo el proceso de diseño, creación y construcción de un simulador para los estudiantes del programa Gestión en la salud y seguridad en el trabajo del Politécnico Grancolombiano, que va desde el análisis de riesgo hasta la proposición de controles para la mitigación. En este capítulo se abordará el proceso elaborado para estos tres riesgos.

\section{Marco teórico}

\section{Problemática que atiende}

Una de las dificultades que se ha encontrado en el programa Profesional de la Gestión en la seguridad y salud laboral en modalidad virtual de la Institución Universitaria Politécnico Grancolombiano, es la falta de espacios de praxis laboral para que el estudiante aplique los conocimientos y conceptos teóricos a través de un proceso aplicado en el sector real. 
Se tiene un espacio de práctica laboral donde el estudiante va a una empresa y realiza todo un proyecto de intervención. La dificultad es que cuando el estudiante atiende este módulo ya está en el último semestre y lo que se evidencia es que no ha tenido la práctica suficiente en su proceso de formación. Por tal razón, se requieren más estrategias que permitan la práctica de los estudiantes y se evidencie el conocimiento adquirido a través del hacer. Para la línea de higiene y seguridad industrial se diseñó la estrategia de aprendizaje donde todos los estudiantes deben realizar la identificación, diagnóstico, análisis, evaluación y mitigación de los riesgos laborales definidos por la Guía Técnica Colombiana (GTC-45) empleando diversas metodologías, para ello se eligieron varios métodos cada uno propio del tipo de riesgo. Dichas metodologías se aplican en cinco tipos de empresas que son: oficina, laboratorio, pozo petrolero, manufactura y servicios generales. El propósito de aprendizaje es que el estudiante apropie una metodología para el análisis de riesgos acorde con la normatividad vigente para el país, esto con el fin de que si el estudiante apropia una metodología podrá aplicarla a cualquier tipo de empresa. El proceso busca desarrollar las habilidades necesarias para que un estudiante pueda realizar el levantamiento de los riesgos laborales y proponga estrategias de mitigación y planes de gestión del riesgo para implementar en la organización.

\section{Antecedentes}

Los simuladores se crean para dar solución a una necesidad generada dentro de las aulas virtuales que es la práctica laboral o la aplicación de los conceptos trabajados en los sílabos de los módulos en escenarios de práctica, por tal razón el proceso inició con la búsqueda en el mercado de un software apropiado que pudieran incluirse en el proceso de aprendizaje de los estudiantes. El software debía cumplir con las condiciones que permitiera al estudiante hacer todo el proceso desde la identificación hasta la mitigación del riesgo. Entre la investigación realizada se encontraron varias herramientas que desarrollan los temas de prevención de riesgos laborales entre ellos:

\section{Simulador virtual de prácticas de evaluación de riesgos laborales,} el cual se ocupa de permitir la observación de las diferentes condiciones de trabajo a través de las cuales se identifican los riesgos y analizan la información dada por el mismo sistema, incluyendo la opinión de los trabajadores. Se puede consultar en: http://www.istas.net/simulador/ 
Dicho simulador presenta la dificultad de no permitir una instalación sencilla lo cual obstaculiza el proceso de trabajar en línea. Por otro lado, si bien brinda los ejecutables a los estudiantes, no posibilita hacerles un seguimiento del cual se pueda obtener la información de su desempeño en la herramienta y de la labor realizada.

Tampoco cubre la totalidad de la temática estudia los riesgos locativos, maquinarias, sustancias químicas y ergonomía, pero no incluye los factores de riesgos biológicos, naturales y físicos. Como está dirigido a técnicos, no cubre los contenidos necesarios para un programa profesional.

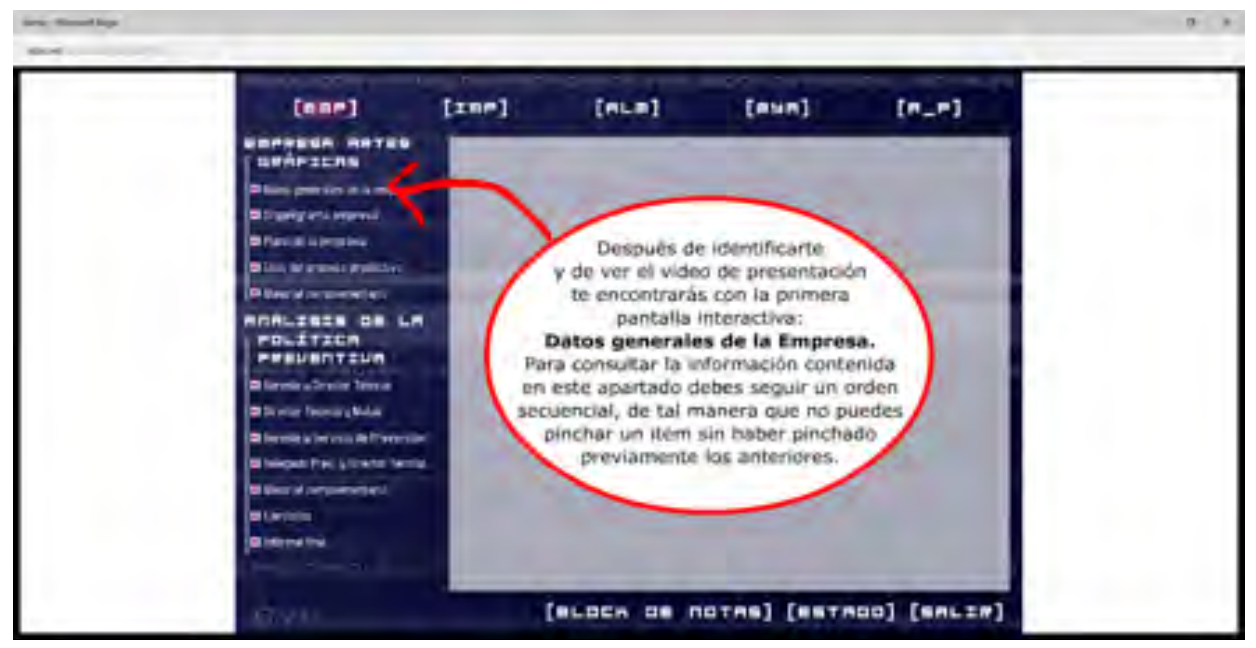

Figura 1. Pantallazo simulador virtual de prácticas de evaluación de riesgos laborales.

Fuente: Istas (2012)

2. Simulador virtual de emergencias y evacuación: Se identificó una segunda herramienta que simula en dos tipos de ambientes diferentes que permiten al estudiante hacer la aplicabilidad conceptual, en este caso, únicamente de atención de emergencias y rutas de evacuación. La herramienta fue descartada porque no aplica a la temática. Se puede consultar en: www.prevencion.ceg.es/modulos/senalizacion

3. Prevención de riesgos laborales permite adquirir hábitos de comportamiento que disminuyan las posibilidades de sufrir un 
accidente en el puesto de trabajo y se enfoca únicamente en el área de la construcción, por este motivo se descarta como temática para los riesgos biológicos y químicos y se centra solo en los de seguridad. Se puede consultar en: http://www.osalan.euskadi.eus/contenidos/ informacion/formacion_aula_virtual/es_aul_virt/adjuntos/Programa_ Seg_Construccion/marcos.htm

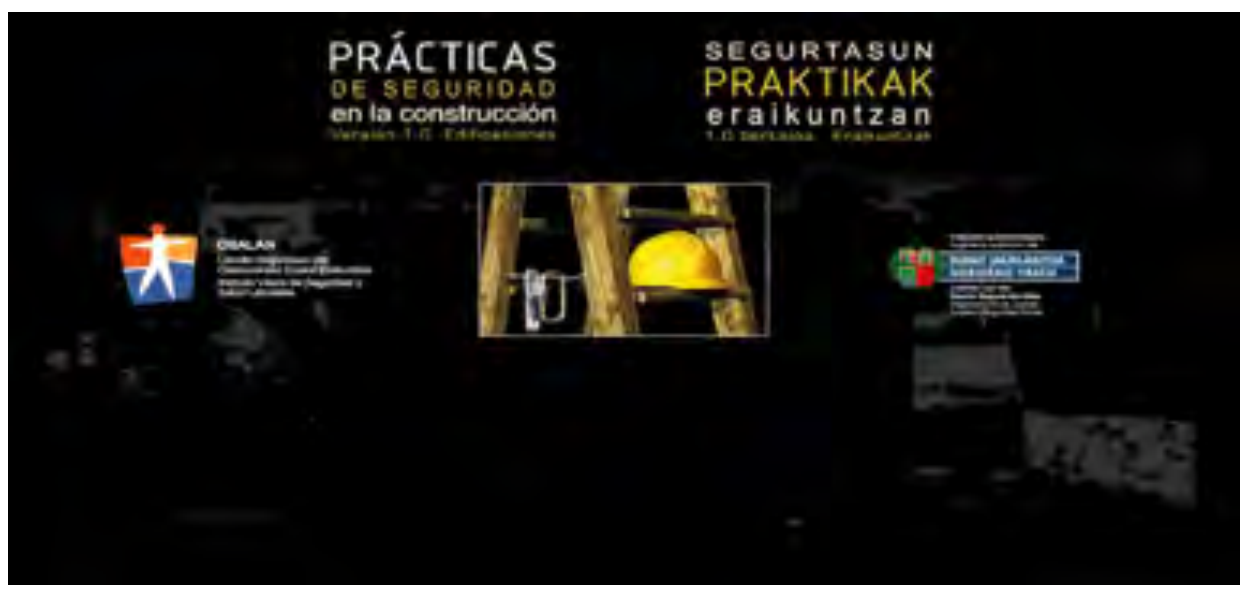

Figura 2. Pantallazo simulador de prácticas de seguridad en la construcción

Fuente: Osalan

4. LUDUS Es un sistema de entrenamiento virtual de personal industrial en la identificación y toma de decisiones relativa a las situaciones de riesgos laborales. El simulador funciona bajo tecnología de realidad virtual con animaciones 3D y con avatares que son los operadores de la empresa experimentan riesgos reales en primera persona, mejorando la percepción sobre la detección de riesgos y una mayor percepción sobre las consecuencias de los mismos.

La simulación proporciona un entorno seguro para la práctica en la toma de decisiones a la hora de atender riesgos. Como se observa en la figura 3.

El simulador emula procesos de forma global y no permite profundizar en un tema específico dentro de los entornos empresariales que ofrece.

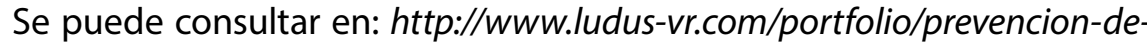
riesgos/ 


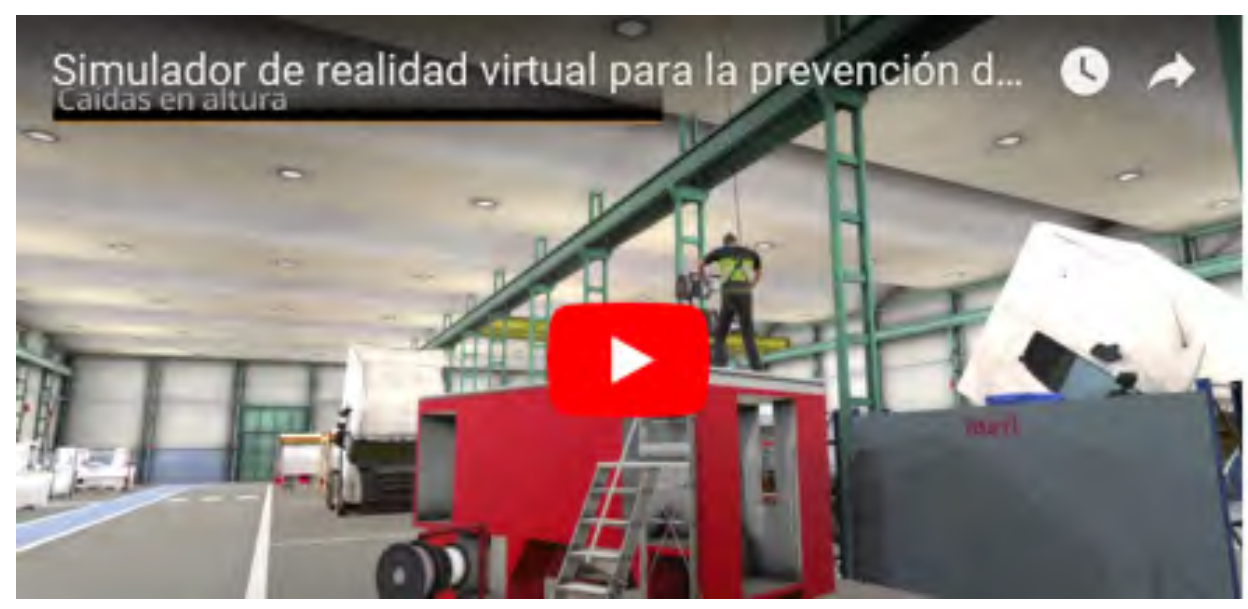

Figura 3. Pantallazo simulador LUDUS

Fuente: Ludus

5. Simulador para la formación en prevención de riesgos: es un entrenamiento que se realiza en espacios reales de manera presencial, no es un simulador como herramienta de software, sino que se simulan situaciones con personas y equipos reales como si estuvieran en una empresa real. El estudiante o practicante va al laboratorio y allí encuentra los equipos y una serie de maniquíes que funcionan como los colaboradores a los cuales el estudiante debe intervenir.

El proceso cuenta con once estaciones de entrenamiento que son: espacios confinados, trabajos en altura, exclusión de materiales extraños, protección contra incendios, protección radiológica, descargos, técnicas de prevención del error humano, izado y movimiento de cargas, riesgo eléctrico, productos químicos, equipos de protección individual y señalización. La figura 4. Muestra algunas de las estaciones. Se trabaja en riesgo químico y de seguridad, no se trata el riesgo biológico. Se puede consultar en: http://www.belt.es/noticiasmdb/HOME2_notaprensa. asp?id $=8959$ 

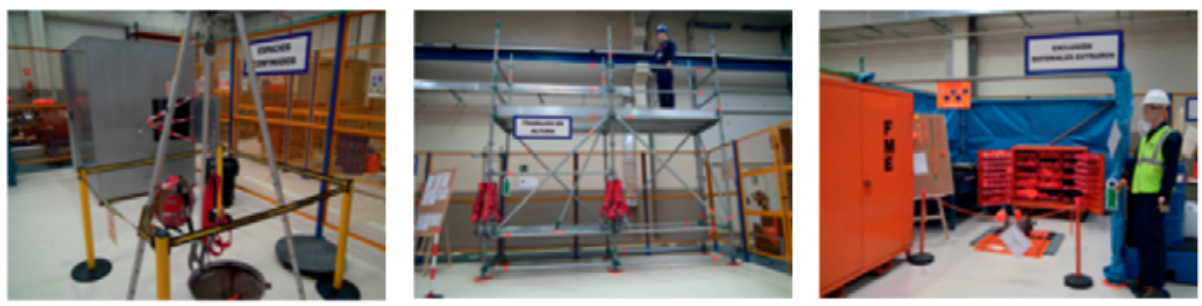

Figura 4. Pantallazo de las estaciones del simulador para la prevención de riesgos.

Fuente: Mapfre

Es una herramienta de práctica interesante para los estudiantes ya que al ser vivencial tiene otros factores motivacionales importantes, sin embargo, es complejo aplicarlo en procesos virtuales porque requiere la asistencia de los estudiantes a tales espacios. Por otro lado, al tener unas estaciones especializadas puede ser más útil para procesos de profundización como líneas de trabajo profesional porque además certifica a los participantes. Por ejemplo, si se trabaja en alturas, la persona queda certificada para el trabajo en alturas, por tal razón no necesariamente debe pasar por todas las áreas para su certificación.

6. Por último, se indagó un portal de juegos interactivos centrados en riesgos laborales, son juegos muy puntuales que sirven como complemento en el módulo, $u$ objetos de aprendizaje para cosas puntuales, pero no como una estrategia que permita evidenciar la actuación del estudiante en escenarios reales. Se puede consultar en: https://higieneyseguridadlaboralcvs.wordpress.com/juegos-interactivos/

De las herramientas indagadas no se encontró ninguna enfocada al riesgo biológico, algunas trabajaban el riesgo químico, y la mayoría se centran en los riesgos de seguridad. Tampoco se identificó una herramienta que siguiera el proceso de identificación, evaluación y control del riesgo, que es primordial con el perfil de los egresados que busca que los estudiantes sean capaces de intervenir una organización en cuanto a salud laboral se refiere, fundamentalmente en lo preventivo y correctivo de los riesgos presentes en condiciones laborales reales. 
La conclusión de la indagación es que los programas o simuladores encontrados son muy básicos para las necesidades formativas que requiere el programa (algunos no se incluyeron en este documento porque no aportaban al mismo), en especial si se habla de riesgos tan específicos como los que se abordan en este proyecto.

\section{Estrategia de aprendizaje}

Para hablar de la estrategia de aprendizaje se deben formular dos parámetros, el primero, las estrategias pedagógicas empleadas que enmarcan el uso de la herramienta. El segundo, la forma cómo se pensó desde lo pedagógico el proceso de simulación para los tres tipos de riesgos.

Como ya se mencionó, está estrategia se basa en el aprender haciendo, porque los métodos de análisis de riesgos laborales ya existen, incluso en este proyecto una de las complejidades para cada riesgo fue seleccionar el método más apropiado que cumpliera las necesidades de las empresas, que fuera acorde con la normatividad legal, fácilmente comprensible y aplicable por el estudiante. Es importante revisar que el proyecto está dirigido a aprendices por lo que debe contemplarse la posibilidad del error y la equivocación; asimismo, las indicaciones y los pasos deben ser muy intuitivos para que el estudiante pueda finalizar el proceso y sentir que aprendió.

No sólo se trabaja el hacer, también se estructura una estrategia donde el simulador no da respuestas, estas dependen de las decisiones, observaciones e indagaciones que hace el estudiante. El simulador le presenta una situación de un contexto real y el aprendiz debe resolverlas. Por lo tanto, se plantea una estrategia de aprendizaje situada que emplea situaciones de la vida real. Según Sagastegui (2004) el aprendizaje situado, al concebir la actividad en contexto como el factor clave de todo aprendizaje, ubica a la educación como parte integrante e indisociable de las diversas prácticas de la vida cotidiana, sólo que aquí son mediadas por una herramienta tecnológica que emula la situación de la empresa, buscando que el aprendizaje sea significativo puesto que aplica sus conocimientos teóricos a una posible situación real que enfrentará como futuro profesional. Stein (1998) se refiere al aprendizaje situado como "una forma de crear significado desde las actividades cotidianas de la vida diaria".

La idea es que por medio del aprendizaje situado entre otras cosas se favorezca el aprendizaje significativo y por descubrimiento. El aprendizaje significativo 
no sólo se construye a través de una herramienta de alta interacción, también se edifica con situaciones relevantes que reten al estudiante en un proceso de análisis. Según Rivas (2007) el aprendizaje significativo toma en consideración tanto los procesos cognitivos del aprendiz como el "mundo" individual de las emociones. Por tal razón el diseño es integral.

Con la estrategia se dio inicio al diseño pedagógico de los escenarios apropiados para los riesgos químicos, biológicos y de seguridad; en seguida, se definió un proceso estandarizado para el análisis, diagnóstico, evaluación y control en los tres tipos de riesgos. Sin embargo, la metodología de evaluación se enfocó a cada tipo de riesgo.

Se pensó en lo concerniente a un resultado para entregar por el estudiante, en un aprendizaje basado por proyectos (ABP). Donde los estudiantes construyen su conocimiento a través de una tarea específica (Swiden, 2013). En este caso el proyecto consiste en elaborar un plan de intervención donde el simulador le muestra la situación, el estudiante identifica los peligros, después los evalúa y de acuerdo con sus resultados construye dicho plan con los diferentes controles para mitigar el riesgo. En el ABP como requisito los estudiantes deben generar un producto, presentación, o ejecución de la solución (Larmer, 2015). En el proceso ese plan de intervención es el producto final, que en la vida real es el informe que entrega el evaluador de riesgos a una organización para que lo implemente en sus colaboradores o sus instalaciones. Los estudiantes trabajan con el proyecto asignado de manera que su abordaje genere productos para su aprendizaje (Moursund, 1999).

Para el proceso de simulación se pensó en una estrategia que permitiera la identificación de un peligro, posterior análisis, diagnóstico, evaluación y control. Esta estrategia se hizo de forma transversal a los tres riesgos, pues los tres métodos de análisis coinciden en que el primer paso es la identificación del peligro implicado, luego viene la valoración, la interpretación de los resultados y las propuestas de control para mitigar el riesgo. En la tabla 1. Se muestran los tres procesos de cada una de las metodologías y se puede observar las coincidencias en sus pasos. 
Tabla 1. Pasos de los métodos de evaluación de riesgos de Seguridad, Químico y Físico.

\begin{tabular}{|c|c|c|c|}
\hline & GTC45 & GATISO DEL BENCENO & BIOGAVAL \\
\hline a. & $\begin{array}{l}\text { Definir el instrumento para } \\
\text { recolectar la información. }\end{array}$ & $\begin{array}{l}\text { Determinación de los } \\
\text { puestos a evaluar. }\end{array}$ & $\begin{array}{l}\text { Determinación de los } \\
\text { puestos a evaluar. }\end{array}$ \\
\hline b. & $\begin{array}{l}\text { Clasificar los procesos, } \\
\text { actividades y tareas. }\end{array}$ & $\begin{array}{l}\text { Identificación del peligro } \\
\text { químico implicado. }\end{array}$ & $\begin{array}{l}\text { Identificación del agente } \\
\text { biológico implicado. }\end{array}$ \\
\hline c. & Identificar los peligros. & $\begin{array}{l}\text { Definiciones y } \\
\text { caracterización de los } \\
\text { solventes. }\end{array}$ & $\begin{array}{l}\text { Cuantificación de las } \\
\text { variables determinantes } \\
\text { del riesgo. }\end{array}$ \\
\hline d. & $\begin{array}{l}\text { Identificar los controles } \\
\text { existentes. }\end{array}$ & $\begin{array}{l}\text { Valoración peligrosidad } \\
\text { Intrínseca de las sustancias. }\end{array}$ & $\begin{array}{l}\text { Medidas higiénicas } \\
\text { adoptadas. }\end{array}$ \\
\hline e. & $\begin{array}{l}\text { Valorar riesgo: } \\
\text { i. Evaluar el riesgo. } \\
\text { ii.Definir los criterios para la } \\
\text { aceptabilidad del riesgo. } \\
\text { iii. Definir si el riesgo es aceptable. }\end{array}$ & Valoración vía de Ingreso. & $\begin{array}{l}\text { Cálculo del nivel de } \\
\text { riesgo biológico (R). }\end{array}$ \\
\hline f. & $\begin{array}{l}\text { Elaborar un plan de acción para } \\
\text { el control de los riesgos. }\end{array}$ & $\begin{array}{l}\text { Valoración de cantidades } \\
\text { usadas. }\end{array}$ & $\begin{array}{l}\text { Interpretación de } \\
\text { los niveles de riesgo } \\
\text { biológico. }\end{array}$ \\
\hline g. & $\begin{array}{l}\text { Revisar la conveniencia del plan } \\
\text { de acción. }\end{array}$ & Medición de las sustancias. & \\
\hline h. & $\begin{array}{l}\text { Mantener y actualizar: } \\
\text { i. Seguimiento de controles. } \\
\text { ii. Aseguramiento de los controles } \\
\text { implementados. }\end{array}$ & $\begin{array}{l}\text { Interpretación de los niveles } \\
\text { de riesgo químico. }\end{array}$ & \\
\hline i. & $\begin{array}{l}\text { Documentar el seguimiento } \\
\text { de la implementación de los } \\
\text { controles. }\end{array}$ & & \\
\hline
\end{tabular}

Fuente: Creación propia tomado de GTC-45, Gatiso de Benceno y Biogaval

Se crearon dos escenarios de práctica, el primero solo aplica para la oficina y es individual, es una simulación guiada por un avatar llamado Bernard (alusión a Bernardino Ramazinni) que brinda las orientaciones, este proceso es autocontenido, es decir, el propio sistema evalúa y retroalimenta las respuestas del estudiante. Esta primera simulación tiene un propósito introductorio y de manejo inicial de la herramienta. Después se hace la simulación grupal con la elección aleatoria de una empresa (laboratorio químico, manufactura de telas, pozo petrolero, servicios generales) que se asigna a un grupo de estudiantes, 
aquí inician la variedad de posibilidades porque el simulador es quien asigna la empresa pues el estudiante debe estar en capacidad de analizar cualquier empresa. Además, cada empresa cuenta con cinco colaboradores y el simulador escoge tres de ellos, generando que se tenga una relación de quince combinaciones diferentes en los personajes. Para completar el caso el simulador tiene siete riesgos y se le asignan cuatro de ellos, significa que se tiene 28 situaciones diferentes. A nivel pedagógico la pretensión es que no existan dos escenarios iguales para dos grupos de estudiantes diferentes para que así los estudiantes enfrenten situaciones distintas de una empresa a otra como sucedería en su quehacer laboral.

Por lo tanto, el primer paso de la simulación es la identificación del peligro, en el simulador se diseñó un escenario en el que es posible visualizar la empresa y con el recorrido del mouse el estudiante identifica, diligencia la primera ficha de información. Posteriormente se define el tipo de riesgo: biológico (agentes), químico (sustancias), seguridad (locativo, mecánico, eléctrico y públicos). En dicha identificación se hace el levantamiento de la información dada por los empleados de cada una de las organizaciones y para cada tipo de riesgo, se diligencian las fichas que le darán al estudiante la información para la evaluación. Con esta información se elabora el diagnóstico inicial.

Posteriormente se ejecuta la valoración del riesgo, para ello se procede a la evaluación del peligro para definir si es un riesgo inminente o no para los empleados. La elección que se realiza de la metodología para cada riesgo depende de los siguientes parámetros: la cantidad de información que se pueda obtener mediante su análisis y aplicación, la practicidad y efectividad (facilidad de aplicación del método y funcionalidad en diferentes tipos de empresas), por último, el diagnóstico y comprobación de los diferentes resultados generados como respuesta de un análisis. Aquí se aplica un método de valoración propio de cada riesgo:

- Biogaval: se utiliza para evaluar el peligro biológico, es aquel relativo a la presencia de un organismo o sustancia derivada del mismo que esboza una amenaza para la salud del trabajador; se genera comúnmente de la exposición a microorganismos que se pueden encontrar en el ambiente $\mathrm{u}$ otros medios de transmisión presentes en las condiciones laborales.

El Manual práctico para la evaluación del riesgo biológico en actividades 
laborales diversas (Biogaval) (2016) publicado por el Instituto Valenciano para la seguridad y salud en el trabajo - INVASSAT, inicialmente en 2004 como complemento al RD 664/97 y a la Guía Técnica del Instituto Nacional de Seguridad e Higiene en el Trabajo en España sobre protección de los trabajadores frente a la exposición a agentes biológicos. La segunda versión se realizó en el 2013 y la más reciente en el 2016, sobre la cual se basó el proceso para el simulador. Esta metodología ha sido adoptada por varios países entre ellos Colombia. La guía es muy versátil porque desde el inicio establece que no existe manera de evitar algunos riesgos, aunque se podrían determinar principios básicos con acciones preventivas que permitieran evaluarlos y controlarlos para minimizar su impacto; es la manera como se procede en las condiciones laborales reales y se recrea en la herramienta de simulación.

Buscando siempre proteger al trabajador de la exposición a agentes biológicos durante el desarrollo de su trabajo, se desarrolla el método que permite hacer la evaluación de riesgo biológico, y así, establecer principios básicos y directrices para tener en cuenta para la consecución del diagnóstico, evaluación y control de los riesgos en cuestión. Esto se fundamenta gracias a la herramienta que permite con pocos pasos que inician con la determinación del puesto o espacio a evaluar, la identificación del agente y cuantificación de las variables determinantes del riesgo. Dentro de estas últimas están la clasificación del daño, vía de transmisión, medidas higiénicas adoptadas, cálculo del nivel del riesgo biológico e interpretación de dichos niveles para la definición de controles.

- Gatiso de Benceno: se utiliza para evaluar el peligro químico, es aquel riesgo susceptible de ser generado por una sustancia química la cual puede tener diferentes vías de ingreso (dérmica, respiratoria, digestiva) y producir efectos agudos o crónicos que generan diferentes enfermedades o intoxicaciones.

La Guía de Atención Integral de Salud Ocupacional Basada en la Evidencia para Trabajadores Expuestos a Benceno y sus derivados (GATISO- BTXEB) (2007) hace parte de las guías de atención integral en Seguridad y Salud en el trabajo aplicadas por el Ministerio de Protección Social en Colombia, fue realizada con el apoyo de la Pontificia Universidad 
Javeriana; esta guía establece técnicas relacionadas con la prevención, diagnóstico y tratamiento de la intoxicación de los trabajadores por el benceno y sus derivados, identifica los factores de riesgos ocupacionales generados a partir de intoxicación por sustancias químicas de cualquier índole, establece los mecanismos para realizar dicho diagnóstico y establece la relación con los diferentes procesos que realiza el trabajador.

- GTC - 45: es utilizado para evaluar el peligro de seguridad. Se relaciona con los accidentes laborales ocasionados bajo circunstancias no adecuadas en las instalaciones (locativo, mecánico, eléctrico y públicos), o de un ambiente de trabajo inconveniente que tenga un riesgo latente y como consecuencia produzca afecciones físicas a los trabajadores.

La Guía Para la Identificación de los Peligros y La Valoración de los Riesgos En Seguridad y Salud Ocupacional (2012), publicada por el ICONTEC y bajo los principios fundamentales de la norma NTC-OHSAS 18001, en el proceso de gestión del riesgo de la norma BS8800 y la NTP330 del Instituto Nacional de Seguridad e Higiene en el Trabajo y la NTC ISO 31000, proporciona las directrices claras para realizar la identificación de los peligros y valorar el riesgo de seguridad con el fin de darle a las organizaciones las herramientas necesarias para identificar dentro de ellas las necesidades en temas de protección y seguridad, para garantizar el buen desarrollo de las labores de los trabajadores.

Continúa la interpretación de los resultados que conllevará a la proposición y debida argumentación de los posibles controles para mitigar el riesgo ya sea en la fuente, en el medio o en el colaborador. A partir de los resultados el aprendiz genera una serie de controles y determina los criterios de selección, este punto de simulación se pensó para que el equipo de estudiantes proponga de forma libre los controles que considere apropiados. Lo fundamental es la justificación como profesional en seguridad y salud en el trabajo de la elección, según sus resultados y su análisis. Esta argumentación es parte del plan de intervención y es evaluada por el tutor.

Algunos métodos proponen el seguimiento y la implementación del plan. La estrategia de aprendizaje está pensada para el diagnóstico, análisis y propuesta de mitigación de un riesgo en una organización. No cubre el proceso posterior 
de seguimiento e implementación del plan de manejo del riesgo, debido a que se requeriría una empresa real donde se evidencie la implementación o cambiar las características del simulador y generar un abanico de controles definidos por el mismo para que puedan evidenciarse en los escenarios (o empresas); sin embargo, ese no es el propósito pedagógico, pues lo que se pretende es que el estudiante sea quien investigue los posibles controles, argumente su elección para dar su postura como profesional en seguridad y salud en el trabajo. Además, se apuesta a la adecuada toma de decisiones, a que visualice el control no sólo desde la mitigación del riesgo sino desde la factibilidad de implementarlo a nivel económico, locativo y de recurso humano. El objetivo es que el estudiante active su creatividad y su sentido común, algo fundamental para el ejercicio profesional que sólo se adquiere mediante la praxis.

\section{Modelo para la simulación}

El proceso para el estudio del riesgo laboral biológico, químico, y de seguridad se realizó en las siguientes etapas que se evidencian en la herramienta:

1. Identificación de peligros: Se hace la determinación de los puestos a evaluar, como ya se mencionó, el simulador crea una situación que se le asigna al estudiante de forma aleatoria, para ello toma cuatro de siete peligros existentes en el entorno laboral de la empresa (oficina, pozo petrolero, laboratorio clínico, manufactura, empresa de servicios), lo que significa que el estudiante tiene la posibilidad de recibir una de veintiocho posibilidades de riesgos diferentes. De igual manera para el análisis del impacto del riesgo en los colaboradores le asigna tres de cinco personajes posibles, lo que significa que puede recibir una de quince posibilidades de combinación de personajes. De esta manera tenemos un abanico de 420 casos posibles, al estudiante se le asigna uno de ellos lo que significa que el espectro de análisis y variedad de casos es significativo para que pueda realizar diversos tipos de simulación. Además, como el análisis se hace a una organización completa, el escenario de simulación se amplía a 1680.

Para realizar la identificación del peligro, existe un supervisor de cada organización que la presenta de modo detallado, así como el proceso productivo que en ella se desarrolla. Las tareas, procedimientos, materias primas utilizadas, equipos de trabajo, trabajadores que se encuentran en 
cada puesto, su estado de salud, edad, sexo y tiempo de exposición las da cada uno de los personajes por medio de una entrevista. De igual manera, el estudiante hace un recorrido por las áreas de la empresa e identifica los posibles elementos peligrosos existentes en el ambiente de trabajo, de esa manera tiene los insumos necesarios para diligenciar la primera ficha que se denomina identificación de peligros.

Después el estudiante continúa con la identificación de los diferentes peligros para ello debe realizar una entrevista a los personajes o trabajadores de la empresa que le asignó el simulador, quienes a través de audios brindan la información para que el alumno diligencie una serie de fichas con los datos del trabajador, del ambiente y de las circunstancias de exposición, de la tarea o actividad. Con la información obtenida se procede a la evaluación del peligro para determinar si es o no un riesgo.

2. Evaluación del riesgo: Se hizo de manera diferente para cada tipo de riesgo por las especificidades de estos, por lo tanto, se empleó una metodología de evaluación para cada uno de ellos (Biogaval - Riesgo Biológico, Gatiso del Benceno - Riesgo Químico y GTC - 45 - Riesgo de Seguridad.

a. Riesgo Biológico: para la evaluación de este tipo de riesgo se utilizó el Biogaval porque es una guía sencilla y clara que en últimas permite orientar al personal encargado del manejo de dichos riesgos, del cómo realizar una prevención priorizando las medidas preventivas que debe adoptar.

Según Llorca J., Soto P., Laborda R., Benavent S. (2013) el Biogaval consta de los siguientes pasos:

i. Determinación de los puestos a evaluar.

ii. Identificación del agente biológico implicado.

iii. Cuantificación de las variables determinantes del riesgo

iv. Medidas higiénicas adoptadas.

v. Cálculo del nivel de riesgo biológico (R).

vi. Interpretación de los niveles de riesgo biológico. 
Ya hemos explicado cómo funciona la determinación de los puestos en la herramienta. Para realizar la identificación del agente biológico implicado, se toma la información dada por el supervisor, los personajes y el análisis del puesto de trabajo.

En cuanto a la cuantificación de las diferentes variables de cada uno de los riesgos identificados dentro del simulador el estudiante e informado por parte del trabajador acerca de las alteraciones de salud que presenta y que están afectando el desarrollo de sus actividades en un área de la empresa previamente definida. Asimismo, recibe información propia del caso como las diferentes vías de transmisión de los agentes y los tiempos de exposición durante las tareas desarrolladas por cada personaje.

La clasificación del daño es determinada por el estudiante de acuerdo con los siguientes factores: la vía de transmisión, por medio del personaje el estudiante debe deducir la vía de ingreso del agente al trabajador; la tasa de incidencia del año anterior de acuerdo con la información del supervisor y de los empleados, que son los datos necesarios para realizar el cálculo de la fórmula. Los empleados manifiestan si han sido vacunados durante la entrevista e informan la frecuencia de realización de tareas de riesgo.

Una de las actividades que se le solicita al estudiante dentro del simulador, pero que debe hacerlo por fuera de este, es el diligenciamiento del formulario con las medidas higiénicas adoptadas que se toman como factores de corrección que serán el insumo para el cálculo de la fórmula empleada por el Biogaval.

Cálculo del nivel de riesgo biológico (R). Una vez el estudiante cuenta con cada uno de los valores que el simulador le solicita de acuerdo con el método, aplicará la formula predeterminada para obtener el cálculo final con el nivel del riesgo. De acuerdo con el valor obtenido, el estudiante debe interpretar su significado y validar sus niveles con el fin de determinar las medidas de prevención pertinentes a cada uno de los factores de riesgo evaluados.

Interpretación de los niveles de riesgo biológico. Finalmente, en este espacio, el estudiante una vez cuenta con el cálculo final del nivel del riesgo y teniendo en cuenta cada factor de riesgo, determina el control a implementar el cual está basado en la información que el estudiante va a encontrar en la GTC-45 con relación a la clasificación de controles. El alumno clasifica el control y argumenta las razones por las cuales eligió ese control para cada uno de los factores de riesgo, Esta justificación es parte del informe final que debe entregar el estudiante al tutor. 
b. Riesgo Químico: Para la evaluación de este tipo de riesgo se utilizó Gatiso del Benceno ya que es una guía sencilla que permite la valoración de las sustancias presentes dentro de un entorno laboral que emite recomendaciones basadas en las evidencias para el manejo integral de las sustancias mediante el desarrollo de la promoción, prevención, diagnóstico, tratamiento y control asociado con la exposición al benceno y sus derivados.

Todo lo anteriormente expuesto, se desarrolla por medio de un proceso sistémico que permitirá al aprendiz la identificación, evaluación y control de la exposición ocupacional a riesgos químicos en ambientes de trabajo como se hace evidente en el desarrollo de la simulación.

Según el Ministerio de la Protección Social y la Pontificia Universidad Javeriana (2007) la Gatiso del Benceno establece los siguientes pasos para la valoración del riesgo químico:

i. Determinación de los puestos a evaluar

ii. Identificación del peligro químico implicado

iii. Definiciones y caracterización de los solventes

iv. Valoración peligrosidad Intrínsecas de las sustancias

v. Valoración vía de Ingreso

vi. Valoración de cantidades usadas

vii. Medición de las sustancias

viii.Interpretación de los niveles de riesgo químico.

La determinación de los puestos ya se explicó cómo funciona en la herramienta. Para realizar la identificación del agente químico implicado, se toma la información dada por el supervisor, los personajes y el análisis del puesto de trabajo. Sin embargo, existe una diferencia con el modelo propuesto por la Gatiso de Benceno, la cual solicita: el inventario de sustancias químicas, el inventario de procesos, la fuerza laboral de circunstancias de exposición y la identificación de medidas de control existentes. El simulador le solicita al estudiante que identifique el peligro o la sustancia, el área de trabajo o proceso, las circunstancias de exposición (cuántos se exponen, por qué, cuándo y en qué condiciones, tareas y turnos en los que ocurre) y las medidas de control existentes. Con 
esta información puede caracterizar el tipo, tiempo, lugar y circunstancia de riesgo que solicita la guía del Benceno.

Según la Gatiso de Benceno, en el inventario de sustancias químicas se solicita identificar la presencia de la totalidad de las sustancias químicas en el lugar de trabajo, en términos de características físicas y químicas, denominación, estado físico, naturaleza toxicológica, etc. Este es el punto que cambia con respecto al proceso de simulación. Aquí el estudiante identifica todas las características de las sustancias, para ello debe investigar las diferentes hojas de seguridad que puede consultar en la biblioteca del simulador. La cantidad de sustancias químicas no se tiene en cuenta porque la metodología del simulador está pensada debido a los efectos de manipular la sustancia en un determinado proceso y no de la cantidad de sustancia existente en el ambiente laboral. Esto no se contradice con la Gatiso de Benceno por el contrario permite al estudiante reconocer los efectos puntuales de la sustancia.

En cuanto a la cuantificación de las diferentes variables de cada uno de los riesgos identificados dentro del simulador el estudiante recibe por parte del trabajador o personaje cuales son las alteraciones a su salud que le están afectando durante el desarrollo de sus actividades en un área de la empresa previamente definida. Asimismo, recibe información propia del caso como las diferentes vías de transmisión de las sustancias y los tiempos de exposición durante las tareas desarrolladas por cada personaje.

Para la definición y caracterización de los solventes es el estudiante quien la determina con los siguientes factores: nombre del peligro o sustancia previamente identificado, proceso en el cual es empleado dicha sustancia lo cual lo deduce el estudiante por la información suministrada por el supervisor y los trabajadores, las circunstancias de exposición información que obtiene durante los audios de los trabajadores y descripción de la empresa por parte del trabajador; por último la identificación de los controles que los realiza para reducir la exposición y su eficacia a las diferentes sustancias.

En cuanto a la valoración de la peligrosidad intrínsecas de las sustancias dentro del simulador el estudiante recibe las diferentes hojas de seguridad de las sustancias, en las cuales debe verificar la severidad del efecto tóxico de la sustancia en función de las cinco categorías existentes ( $A, B, C, D$ y E), estas deben figurar en la etiqueta del producto o la hoja de seguridad. 
Valoración vía de Ingreso. Una vez el estudiante cuenta con el nombre de la sustancia puede definir cuál fue la vía de ingreso de acuerdo con la información de los diferentes audios o de la misma hoja de seguridad de la sustancia química la cual encuentra en la biblioteca del simulador.

Valoración de cantidades usadas. Las hojas de seguridad de las sustancias contienen una descripción detallada de la presentación en la que viene la sustancia y los personajes durante los audios hacen mención de las cantidades empleadas en cada proceso.

Medición de las sustancias. En este apartado el estudiante determina el valor umbral límite de las sustancias químicas, la concentración máxima permitida para exposición de trabajadores dentro de su área de trabajo; generalmente se informa en partes por millón (ppm) o en $\mathrm{mg} / \mathrm{m} 3$. Para ello identifica la presentación de la sustancia química, mediante la medición con la bomba define la cantidad de la sustancia para determinar el valor del TLV. Con la medición se obtienen los TLV necesarios para el análisis y determinación del nivel de afección de la sustancia al trabajador. El simulador emula la medición de los TLV con estos valores el estudiante debe contrastar con los valores definidos con cada una de las hojas de seguridad de las sustancias para argumentar los controles que decide sugerir en el informe y decidir si estos son preventivos o correctivos de acuerdo con esa cantidad.

Grupos de exposición similar subjetivos. No aplica al modelo de simulación ya que el abordaje que se realiza de los grupos expuestos es trabajado en un área puntual de la empresa y no se tiene en cuenta la totalidad de esta.

Si la calificación de exposición no es concluyente la Gatiso sostiene que se debe realizar el siguiente análisis: Métodos y técnicas de muestreo, representatividad estadística, grupos de exposición similar objetivos, TLVs y BEls. Esta parte no aplica porque todo el diseño del modelo de simulación está encaminado a que la exposición sea concluyente para facilitar la apropiación de la metodología porque el perfil profesional del estudiante no se enfoca a que él realice el análisis de muestreo y representatividad, sino que interprete adecuadamente los resultados de una medición previa. De esa manera, el alumno puede concluir los niveles confiables y adecuados la exposición del trabajador.

Interpretación de los niveles de riesgo químico. El estudiante interpreta y propone los controles a implementar según los niveles de exposición del colaborador con respecto a la sustancia que este manipula. Tales controles los propone el estudiante como ya se trató en este documento. 
c. Riesgo de Seguridad: Para la evaluación de este tipo de riesgo se utilizó laGTC45, esta guía permite identificar, valorar y corregir los diferentes peligros que hacen parte de los procedimientos, para así, definir unos controles que permitan generar dentro de los procesos acciones preventivas o correctivas en las empresas.

Según el Instituto Colombiano de Normas Técnicas y Certificación (ICONTEC). (2010) la GTC45 establece los siguientes pasos para la valoración del riesgo de seguridad:

i. Definir el instrumento para recolectar la información.

ii. Clasificar los procesos, las actividades y las tareas.

iii. Identificar los peligros.

iv. Identificar los controles existentes.

v. Valorar riesgo

i. Evaluar el riesgo.

ii. Definir los criterios para la aceptabilidad del riesgo.

iii. Definir si el riesgo es aceptable.

vi. Elaborar un plan de acción para el control de los riesgos.

vii. Revisar la conveniencia del plan de acción.

viii.Mantener y actualizar:

ix. Seguimiento de controles

$\mathrm{x}$. Aseguramiento de los controles implementados

xi. Documentar el seguimiento a la implementación de los controles

La guía establece que las organizaciones deberían contar con una herramienta para consignar de forma sistemática la información proveniente del proceso de la identificación de los peligros y la valoración de los riesgos, la cual debería ser actualizada periódicamente (GTC-45). Este documento se hace a través de las fichas que lleva incorporadas el simulador y que se publican en pdf cuando el estudiante solicita el informe de simulación. 
Clasificar los procesos, las actividades y las tareas. Se realiza en la ficha 1 y 2 del simulador que se diligencia con la información del supervisor y los personajes. El estudiante debe consignar el peligro, la descripción del mismo según GTC y la caracterización (descripción de las características y efectos del peligro identificado).

Identificar los peligros. Para ello el alumno puede hacer un recorrido por las instalaciones o escenarios que representan la empresa y detectar los peligros. De esa manera diligencia la ficha 1.

Identificar los controles existentes. La guía propone relacionar todos los controles que la organización ha implementado para reducir el riesgo asociado a cada peligro, esto lo hace a través de la información que provee el supervisor y los colaboradores.

Evaluar el riesgo. Se debe calificar el riesgo asociado, los controles, así como la probabilidad y las consecuencias si éstos fallan. Para ello se solicitan tres procesos:

a. Evaluar el riesgo: el estudiante califica el riesgo asociado de acuerdo con los peligros identificados y la información obtenida de los colaboradores.

El estudiante realiza los cálculos de los siguientes parámetros:

Nivel de deficiencia (ND): es la magnitud de la relación entre el conjunto de peligros detectados y su relación causal directa con posibles incidentes y con la eficacia de las medidas preventivas existentes en el lugar de trabajo (GTC - 45, 2012).

Nivel de exposición (NE): es la situación de exposición a un riesgo que se presenta en un tiempo determinado durante la jornada laboral.

Nivel de probabilidad (NP): es el producto del nivel de deficiencia por el nivel de exposición.

Nivel de consecuencia (NC): es una medida de la severidad de las consecuencias.

Nivel del riesgo (NR): es la magnitud de un riesgo resultante del producto del NP por NC.

b. Definir los criterios para la aceptabilidad del riesgo: esto lo hace después de los cálculos de los diferentes niveles y de 
diligenciar la matriz de riesgos. Aquí el estudiante analiza los resultados obtenidos e interpreta según los lineamientos de la GTC-45 para posteriormente argumentar sí es un riesgo o no.

c. Definir si el riesgo es aceptable: Basado en la interpretación y con los parámetros determinados dentro de la guía técnica el estudiante argumenta el nivel de aceptabilidad de los riesgos para planificar los posibles controles de S y SO.

Elaborar un plan de acción para el control de los riesgos. Con la evaluación y la aceptabilidad de los riesgos los estudiantes proponen los diferentes tipos de control y los clasifica según la GTC-45. Los controles propuestos tienen dos finalidades, la primera mejorar los controles existentes si es necesario, o atender cualquier otro asunto que lo requiera.

Y la segunda, proponer nuevos controles si la empresa no cuenta con ellos. Estos controles pueden ser en la fuente, el medio o el colaborador y pueden ser de sustitución, eliminación, administrativos, de ingeniería y equipos de protección personal (epp). El estudiante puede proponer cualquier tipo de control de los anteriormente mencionados, lo importante aquí es que los justifique de manera apropiada.

La Guía Técnica Colombiana propone tres pasos más que son los siguientes (GTC- 45, 2012):

- Revisar la conveniencia del plan de acción. Revalorar los riesgos con base en los controles propuestos y verificar que los riesgos serán aceptables.

- Mantener y actualizar:

i. Realizar seguimiento a los controles: Se deben llevar seguimientos nuevos y existentes para asegurar que sean efectivos.

ii. Asegurar controles: Certificar que los controles implementados sean efectivos y que la valoración de los riesgos este actualizada.

- Documentar el seguimiento a la implementación de los controles. Establecer el plan de acción que incluya responsables, fechas de programación, ejecución y estado actual, como parte de la trazabilidad de la gestión en S y SO. 
Estos pasos no se tienen en cuenta para el proceso de aprendizaje por varias razones: la primera es que es un proceso de diagnóstico, y como ya se explicó anteriormente, va hasta la proposición de los controles. La segunda es que para hacer un proceso de seguimiento se requiere de una mayor extensión en el tiempo y debemos tener en cuenta que el módulo es de ocho semanas. La tercera, podría implementarse en una segunda versión del simulador, pero está sujeto a cambios o reestructuración curricular del módulo, porque todos los procesos de simulación del Laboratorio de Innovación Educativa de Educación Virtual se hacen de acuerdo con los requerimientos de los programas académicos.

3. Controles: Para los tres simuladores el estudiante debe proponer diversos tipos de controles, clasificarlos y argumentar las razones por las cuales seleccionó dichos controles, se pueden proponer controles que impacten al colaborador, al medio o a la fuente como ya se mencionó. La prevención técnica apropiada (fuente o en el medio), las medidas de control sobre el trabajador que pueden ser técnicas o de ingeniería, las administrativas y la protección personal (EPP). También se consideran en el modelo las medidas de control en formación e información a través de capacitaciones que permitan hacer un proceso de retroalimentación con la empresa. Esto genera un abanico de opciones muy amplio y depende del análisis de cada individuo las decisiones que tome y sugerencias que haga a la empresa. Se proporciona un espacio de escritura para que el estudiante redacte y argumente las decisiones que toma al seleccionar y sugerir a la empresa un control determinado que sea pertinente para la mitigación del riesgo en la misma.

\section{Cómo se elaboró la experiencia}

Como respuesta a una necesidad que se evidenció en los estudiantes actuales y buscando un acercamiento con la realidad laboral a la cual se va a enfrentar el futuro egresado del programa de profesional en Gestión de la Seguridad y Salud Laboral de modalidad virtual del Politécnico Grancolombiano se realiza una experiencia de aprendizaje que propende evidenciar el conocimiento teórico a nivel praxeológico.

Por las especificidades del programa es necesario que la estrategia esté inmersa en un entorno virtual que brinde todos los ambientes, los personajes 
y las herramientas necesarias para que pueda simular las condiciones laborales asociadas a los riesgos biológicos, químicos, de seguridad que pueda encontrar en un ambiente laboral determinado. En este ambiente el estudiante debe tomar decisiones frente a las circunstancias dadas y que vayan a la par con las temáticas y contenidos trabajados en los módulos.

Este trabajo comienza por la construcción de los mapas de funcionamiento del simulador, para ello se descompuso la competencia entre los diferentes factores: estudiante, tutor, simulador, y se evidenció cómo cada uno de ellos tendría diferentes elementos de competencia. Con estos elementos se diseñaron las funciones, es decir, qué haría cada factor; y con esos insumos se construyó el diagrama de relaciones o conceptual del simulador.

Del diagrama se sacó la escaleta que daría la pauta para el desarrollo de todos los procesos internos del simulador; luego se inició con la elaboración de los guiones, para ello se identificaron los aspectos más relevantes para tener en cuenta como cargos de los trabajadores, tipos de empresa, condiciones laborales, ambientes de trabajo, materiales y herramientas, tareas, ubicación geográfica y condiciones generales de la empresa. También se definieron los agentes biológicos y enfermedades laborales que se presentan durante el desarrollo de las diferentes actividades en cuanto al riesgo biológico. Para el riesgo químico se caracterizaron las sustancias e identificaron las posibles enfermedades causadas por la exposición a las mismas. En cuanto al riesgo de seguridad se valoraron las instalaciones locativas y los posibles accidentes de trabajo que se podían generar dentro de las mismas debido a los peligros inminentes.

Con los factores de riesgo definidos para los tres simuladores se prosigue con la identificación de los diferentes escenarios que reunieran las características más sobresalientes con relación a los diferentes agentes biológicos presentes en la zona, sustancias químicas empleadas en los procesos y posibles accidentes que se puedan presentar en cada zona de trabajo.

En seguida, se realizó la escogencia de cada una de las herramientas de análisis para cada tipo de riesgo y se estableció una metodología para el análisis de forma separada para cada tipo de riesgo. De igual manera, se crearon las instrucciones para que el estudiante pueda aplicar el análisis del peligro que hacen parte de cada tipo de riesgo, con el fin de dar al estudiante una cercanía con las condiciones laborales reales encaminadas al cumplimiento de las regulaciones nacionales al respecto.

El estudiante debe ir de la identificación, al análisis, evaluación, interpretación 
y control de los riesgos biológicos, químicos y de seguridad; para ello, se establecieron las características y condiciones de las empresas como: áreas, cantidad de trabajadores, descripción de zonas, tareas y actividades de los trabajadores.

Con todo este insumo se inició la elaboración de los guiones y los escenarios. Se comenzó con la revisión de las condiciones de cada sitio de trabajo para definir los parámetros de estas y su aplicabilidad. Se redactaron los guiones para cada empresa por separado y se hicieron ajustes de acuerdo con el proceso de aprendizaje a seguir dentro del simulador (identificación, análisis, evaluación, interpretación y control). Esta apropiación de una metodología será el componente clave para poder realizar un óptimo proceso en una empresa real como parte de su quehacer diario en su ejercicio profesional.

En los guiones se trató de plasmar las situaciones más relevantes a las que se verá enfrentado el estudiante cuando deba identificar los riesgos biológicos, químicos y de seguridad, con los desencadenantes de estos durante la simulación y el trabajo que deben entregar.

Al realizarse la verificación de los guiones fue notoria la necesidad de ajustarlos puesto que no había una correspondencia directa entre la información que se le brindaba al estudiante desde el simulador y lo que se pedía desde la metodología de análisis (Biogaval, Gatiso de Benceno, GTC-45), esto provocó más de dos meses de imprevistos para el inicio de la programación, pues se hicieron varios ensayos y errores que si se llevaban a programar ocasionarían fallas en el sistema y afectarían la percepción del estudiante. Para encontrar la solución se hizo una observación desde la perspectiva del estudiante, así como emplear personas externas que leyeran los guiones y nos dieran su interpretación para ver si correspondía con lo que estaba escrito. Fue un proceso desgastante pero útil para realizar ajustes y entregar a programación.

Parte de estos ajustes fue caracterizar de nuevo y describir a los personajes que se van a ubicarse en cada área de cada empresa, en algunos casos se tuvo que alterar su caracterización física, mental y psicológica para que así pudiesen dar información pertinente para la empresa, su labor y sus posibles afecciones a la salud; información totalmente necesaria para hacer el proceso de evaluación y análisis.

En cuanto al diseño se crearon los escenarios bases en 3D, que son los escenarios de la empresa, se montó uno por cada empresa, es decir cinco escenarios base en los cuales se ubicaron los riesgos y los colaboradores, es decir, por cada riesgo quedó un escenario con cinco colaboradores y siete riesgos, tanto 
los colaboradores como los objetos donde se desprendían los peligros se renderizaron en 3D. Los cinco colaboradores son transversales a las empresas y los riesgos, exceptuando el riesgo químico que requirió un colaborador para cada riesgo ya que el peligro va directamente relacionado con el contacto a la sustancia. Cada empresa cuenta con un supervisor que la presenta, también describe los procesos de cada área de trabajo para que el estudiante realice un análisis inicial. Para cada colaborador se realizaron los audios, los cuales cambiaban para cada empresa por sus labores o cargos y por el cambio en los peligros, esto implicó la elaboración, grabación y animación de 75 audios, sin contar los audios de los supervisores que son cinco, adicionales a los de Bernard.

Para la ejemplificación de cada peligro se realizaron animaciones 2D donde un personaje genérico mostraba lo que pasaba, por ejemplo, dos cables eléctricos descubiertos que generaban un corto y de allí se genera un incendio, para cada una de estas situaciones se realizaron animaciones para que el estudiante visualizara lo que sucede. Para esto se realizaron 140 animaciones.

\section{Resultados obtenidos}

El resultado más importante es el simulador que está en verificación para su posterior implementación en las aulas virtuales. Se aplicarán estrategias de simulación para los módulos Higiene y Seguridad Industrial I y II. A su vez los contenidos desde el sílabo se ajustaron y se actualizaron de acuerdo con el proceso de escenarios para que fueran consecuentes con el proceso de simulación, ayudando así al mejoramiento curricular.

La herramienta está estructurada a la par con los contenidos abordados en los diferentes módulos que tienen la simulación como estrategia pedagógica. Esto genera que el estudiante durante el transcurrir de su carrera se acerquen o tengan una proyección clara en el ámbito laboral.

Por medio del simulador, se logra que el estudiante aprenda a formular propuestas de intervención orientadas a solucionar problemas presentes asociados a la seguridad y salud en el trabajo.

Tanto docentes como estudiantes al contar con una herramienta de práctica, podrán mejorar la calidad de vida de los trabajadores y el incrementar la productividad de las empresas en temas de Seguridad y Salud en el trabajo. 
El aporte significativo para los tutores en el papel de autores va más allá de la construcción de la herramienta; sirve para enriquecer el conocimiento sobre las temáticas, aterrizar los conceptos abordados en los módulos y llevarlos a una praxis en el estudiante. En la construcción de los guiones y la comprobación de estos fue fundamental ubicarse en el papel del estudiante, esto permite otra perspectiva pedagógica como tutores.

Desde la tutoría el estudiante trabaja la parte conceptual y se espera que apropie dichos conceptos, en cambio con el simulador los estudiantes evidencian tal apropiación para facilitar el trabajo del tutor. Ya que el simulador estimula la interacción continua entre tutor - estudiante y estudiante - estudiante, puesto que depende de un proceso que va de la mano con el desarrollo del módulo.

\section{Conclusiones}

Durante la construcción de estas tres herramientas de aprendizaje el objetivo en común se fundamentó en implementar una herramienta que de manera práctica le permita al estudiante identificar de forma práctica los diferentes métodos existentes para la identificación y valoración de los riesgos biológicos, químicos y de seguridad de tal modo que les garantice adquirir habilidades que les permitan tomar decisiones e influir positivamente dando soluciones pertinentes dentro de los diferentes ambientes laborales expuestos por el simulador.

Con los datos y posibles situaciones o circunstancias generados en el simulador, se puede realizar un análisis claro y conciso que tiene como único fin conocer y manejar los métodos necesarios para la identificación y valoración de los diferentes riesgos.

El simulador es una experiencia de aprendizaje que acerca al estudiante a escenarios reales generándole aportes significativos en el área practica de las temáticas trabajadas a lo largo de la evaluación de los tres riesgos analizados. Entonces, con el simulador tenemos un análisis con valoraciones cualitativas y cuantitativas fundamentales para su desempeño como profesional en el área.

La construcción de un simulador cambia la perspectiva como docente, abre la posibilidad de realizar materiales pensados desde la competencia y como lograr estrategias efectivas que permitan evidenciar la adquisición de estas. Eso implica cambiar la forma como pretendemos llegar a los estudiantes, por ende, un cambio de pensamiento como tutores.

Es un reto para los tutores actuales y futuros construir ambientes virtuales apropiados para el aprendizaje, no es solo una serie de conceptos a tratar, 
sino un proceso que evidencie la adquisición y apropiación del conocimiento, además, que sea capaz aplicarlo en diversas situaciones, esto es uno de los grandes aportes que quedan para los autores quienes pueden desarrollar otras experiencias similares o ampliar la presente.

\section{Bibliografía o referencias}

Baraza, X., Castejon, E., \& Guardino, X. (2014). Higiene Industrial. España: UOC.

Falagan. (2009). Higiene industrial aplicada. España: Fundación Luis Fernando

Larmer, J. (2015). Project-Based Learning vs. Problem-Based Learning vs. X-BL. Recuperado de: http://www.edutopia.org/blog/pblvs-pbl-vs-xbl-john-larmer

Llorca J., Soto P., Laborda R., Benavent S. (2013) Manual práctico para la evaluación del riesgo biológico en actividades laborales diversas - BIOGAVAL. Centro Territorial de Valencia del INVASSAT, Valencia. Recuperado de: http://www.higieneambiental. com/sites/default/files/images/pdf/biogaval2013.pdf

Ministerio de la Protección Social y Pontificia Universidad Javeriana (2007) Guía de Atención Integral de Salud Ocupacional Basada en la Evidencia para Trabajadores Expuestos a Benceno y sus derivados (GATISO-BTX-EB). Dirección General de Riesgos Profesionales y Subcentro De Seguridad Social Y Riesgos Profesionales, Bogotá.

Moursund, D. (1999). Project-based learning using information technology. Eugene, OR, EUA: International Society for Technology in Education. En: Observatorio de Innovación Educativa (2016). Aprendizaje basado en retos. Tecnológico de Monterry. Recuperado en: https://observatorio.itesm.mx/edutrendsabr

Rivas, C. (2007). Organización del conocimiento para un aprendizaje significativo. Saber. Revista Multidisciplinaria del Consejo de Investigación de la Universidad de Oriente, 19 (2), 210-219.

Sagastegui D. (2004) Una apuesta por la cultura: el aprendizaje situado. Sinéctica, Revista Electrónica de Educación. Recuperado de: https://www.ucm.es/data/cont/ docs/1010-2015-05-24-Del\%20aprendizaje\%20difuso\%20al\%20aprendizaje\%20 situacional.pdf

Stein D. (1998) “Situated learning in adult education". En: ERIC Digest, núm. 195.

Swiden, C. L. (2013). Effects of challenge based learning on student motivation and achievement (Tesis de Maestría). Montana State University. Recuperado de: http:// scholarworks.montana.edu/xmlui/handle/1/2817

Velasco. Guía Técnica Colombiana GTC 45. 


\section{Referencias de figuras:}

Istas (2012) simulador virtual de prácticas de evaluación de riesgos laborales. [Pantallazo] Recuperado de: http://www.istas.net/simulador/

Mapfre (2012) Simulador para la formación en prevención de riesgos. Recuperado de: http://www.belt.es/noticiasmdb/HOME2_notaprensa.asp?id=8959

Osalan - Euskadi (2017) simulador de prácticas de seguridad en la construcción. [Pantallazo] Recuperado de: http://www.osalan.euskadi.eus/contenidos/ informacion/formacion_aula_virtual/es_aul_virt/adjuntos/Programa_Seg_ Construccion/marcos.htm

Portafolio (2017) Pantallazo simulador LUDUS. [Pantallazo] Recuperado de: //www.ludusvr.com/portfolio/prevencion-de-riesgos/ 